\title{
Snakes move their scales to increase friction
}

\author{
Hamidreza Marvi ${ }^{\mathrm{a}}$, James P. Cook ${ }^{\mathrm{b}}$, Jeffrey L. Streator ${ }^{\mathrm{b}, *}$, David L. Huc, ${ }^{\mathrm{c}, *}$ \\ ${ }^{a}$ School for Engineering of Matter, Transport and Energy, Arizona State University \\ ${ }^{b}$ School of Mechanical Engineering, Georgia Institute of Technology \\ ${ }^{c}$ Schools of Mechanical Engineering, Physics, and Biology, Georgia Institute of Technology
}

\begin{abstract}
Snakes can climb a range of surfaces, from tree trunks to brick walls, using a hitherto poorly understood mechanism. The bellies of snakes are covered in a series of flexible scales that can be activated by the snake to prevent sliding. It is previously shown that conscious snakes can use this ability to double their friction coefficient relative to unconscious snakes. In this combined experimental and theoretical study, we give further evidence that snakes actuate their belly scales. We perform experiments where we slide snakes backwards atop an array of pillars. Our theoretical model suggests snakes that do not apply an opening moment to their scales should have quite short contact with these pillars. In our experiments, snakes slide their ventral scales down the pillars, prolonging contact. Our modeling suggests that this phenomenon can only occur if snakes apply a moment at the scale base. We hope this result encourages further research in actively-generated friction.
\end{abstract}

Keywords: contact mechanics, friction, flexible scales, active control

\section{Introduction}

Snakes are one of the few organisms that propel themselves by sliding their bodies against the ground. Compared to limbed organisms, this mode seems like a poor locomotory choice. However, snakes are able to move their limbless

\footnotetext{
* Corresponding author

Email addresses: streator@me.gatech.edu (Jeffrey L. Streator), hu@me.gatech.edu (David L. Hu)
}

Preprint submitted to Journal of Biotribology

November 4, 2015 
bodies across a range of terrain, including rock, sand, mud, and leaf litter. Moreover, they can propel themselves both across flat ground and straight up trees and brick walls. Clearly, such versatility suggests they have adaptations to both reduce and increase friction as they move. However, most studies of friction cannot be directly applied to understand snake movement. Most friction studies rely on flat or gently rounded surfaces in contact, such as tires and ball bearings. In contrast, biological surfaces such as snakeskin can be scaly, involving consideration of soft flexible components jamming or in stick-slip. Little work has been done on understanding the frictional properties of such surfaces and how they are adapted for increasing friction. The goal of this study is to understand how snakes can resist sliding backwards.

Snakeskin has attracted a great deal of attention for its unique frictional properties [1, 2, 3, 4, 5. Their ventral scales in particular have attracted many attempts to characterize their frictional properties. Most studies involve deceased or unconscious snakes [1, 2, 3, 4, 5, 6, 7,. Classical studies of snake locomotion by Gray involve measurement of the frictional properties of snakes sliding on different substrates such as wood and metal [6, 8]. Gray was the first to observe the frictional anisotropy of the snakeskin, the variation in friction coefficient $\mu$ according to sliding direction. Friction coefficient $\mu$ is the ratio between sliding friction force and normal force. As shown in later experiments 25 by $\mathrm{Hu}$ et al, snakes have the lowest friction coefficient if sliding in the forward direction, twice the friction in the lateral direction and four times the friction in the rearward direction [9]. This anisotropy is due in part to the macro-scale structure of the snake's ventral scales, which resemble overlapping shingles.

One source of frictional anisotropy is microscopic features on the ventral 30 scales. Hazel et al discover pawl-like microscopic features on boa scales [2]. Using atomic force microscopy, they report that tail-to-head friction coefficient of a single scale is 3-4 times that of the opposite direction [2]. Gorb et al study the micro-ornamentation and frictional properties of various parts of the snakes, including dorsal, lateral, and ventral regions [4]. They report ventral 35 scales have the highest frictional anisotropy of $26 \%$, compared to only about 
4-5\% for both lateral and dorsal. These characteristics provide further evidence of a unique specialization of the ventral scales towards friction enhancement in locomotion. Frictional properties of snakeskin are also studied by Abdel-Aal et al [7. Studying the shed skin of a Python regius, the research maps out 40 coefficients of friction for scales on all parts of the body: lateral, dorsal, and ventral. They find frictional anisotropy is more significant in the ventral scales than any other part of the body [7].

Marvi et al report that the friction coefficients of conscious corn snakes are almost twice as those of unconscious snakes implying the importance of active mechanisms [8]. They report experiments qualitatively showing the snake opening and closing its belly scales [8, 10, 11. However, how such motion increases frictional anisotropy remains unknown.

In this study, we investigate how snakes use their scales to resist sliding. In $\S 2$, we describe a new method for recording the force and displacement of a snake scale during sliding tests. We present in $\S 3$ a theoretical model for predicting the frictional force of a single snake scale. In $\S 4$, we present evidence, based on our model predictions, that snakes actively apply an opening moment to their scales to prevent sliding. In $\S 5$, we give suggestions for future work.

\section{Methods}

\subsection{Animal care}

Two juvenile corn snakes Pantherophis guttatus (Fig,1 1 a, b) used in this experiment are cared for in captivity for the duration of our experiments $(N=2$, $m_{1}=0.73 \mathrm{~kg}, m_{2}=0.85 \mathrm{~kg}, L_{1}=131.3 \mathrm{~cm}$, and $\left.L_{2}=139.5 \mathrm{~cm}\right)$. The snakes are fed weekly and housed in separate terrariums with controlled temperature and humidity conditions.

\subsection{Friction measurement}

We designed a device to measure the resistive force of a single snake scale while permitting visualization of the snake scale. The device consists of a series 
of vertical pillars of plastic film of thickness $0.12 \mathrm{~mm}$ transparency sheet $(3 \mathrm{M}$ Dual Purpose CG5000 transparency film) cut with a laser into dimensions 24.2 $\mathrm{mm}$ by $6 \mathrm{~mm}$. We refer to this transparency sheet hereon as "plastic". The array of pillars is then inserted into $2 \mathrm{~mm}$ deep grooves laser cut into a block of wood. Openings on the side allowed us to see the deflection of the entire pillar, which we through our theoretical model, used to predict force. Spacing between pillars of plastic film is chosen to reduce interaction between pillars but facilitate tripping of scales as the snake is pulled across the apparatus.

We use two corn snakes in this study and conduct 10 trials with each snake being conscious and unconscious. In the experiments, a snake is pulled manually over the film array with its ventral scales catching and displacing the pillars

75 (Fig. 1 a, b). We hold the snake with two hands, supporting its body weight such that its ventral surface grazes the tops of the pillars, as would be the case if the snake were climbing vertically. Sliding backwards in this case corresponds to sliding downwards while climbing which happens when the maximum load scales can support is exceeded. In this study, we are interested in investigating this load limit for an individual scale. We test ventral scales located within $\pm 10 \%$ from the midpoint of the snake. All experiments are filmed from the side using a high definition digital video camera (Sony HDRXR200) at 30 frames per second. Image analysis via the open source Tracker is used to record parameters such as scale base and tip positions, scale base angle, and pillar tip position. Since we can precisely track these parameters, we can simulate any of the experimental trials regardless of their variability. However, using the side views we make sure we omit the trials in which the snake belly pushes on the pillars (rather than only tip of ventral scales touching the pillars).

\subsection{Measurement of snake scale}

Our theoretical model requires the shape of the snake scale, its material properties and frictional properties. We discuss measurement of each in turn. All measurements are performed on ventral scales of unconscious snakes. The scale width and length are measured with calipers (Fig, 2 ). The thickness 
and radius of curvature of the scale tip are obtained from scanning electron microscopy (SEM) (Fig,2b). We use Matlab to best fit a circle to the crosssection of the scale tip in the SEM image to determine its radius of curvature.

We measure the elastic modulus of the pillar using DDL Instron tensile test machine according to the Tensile Testing of Thin Plastic Sheeting standard, ASTM D 882. However, we are unable to measure the elasticity of the snake scale. Benz et al use microindentation to measure Young's modulus for ventral scales of a California King Snake. They report a stiffness of $413 \mathrm{MPa}$ for ventral scales of this snake [5]. Using this value as an estimate, we select a value of 400 MPa for the elastic modulus of the corn snake ventral scale.

Friction between snake scale and pillars is measured using an inclined plane experiment. We place the snake on a $2 \mathrm{~m}$ x $1 \mathrm{~m}$ plate covered with transparency. Tilting the plane increases the static friction force felt by the object. The inclination at which the snake begins to slide down the slope reflects the static friction coefficient of that object on the substrate. The details of this experiment are discussed by Marvi et al [8]. We use this technique to measure the friction coefficient between both snake and plastic (3M transparency sheet). We also measure the friction coefficient between glass and plastic.

\section{Model}

\subsection{The snake scale}

The snake scale is modeled as a series of segments, as depicted schematically in Fig.1. The segments are pinned to each other lengthwise. In addition, each segment is coupled to each of its two neighbors by a torsional spring. Each torsional spring exerts an opposing moment in proportion to the relative angle of the connected segments. Additionally, linear viscous damping is imposed on the torsional motion of the segments whereby an opposing moment is exerted about the center of mass in proportion to the angular velocity of the segment. Typi-

cally, we assume damping coefficients for the scale and pillar that correspond to damping ratios of 0.01 and 0.1 , respectively. The damping ratios were selected 
in a heuristic fashion. We chose the smallest values of damping ratios for which high frequency vibrations tended to die out quickly during the simulation. No effort was made to measure the damping characteristics of the snake scale or the pillar. As tabulated in Table1, the snake scale is tapered, and we represent this shape using segments with differing thickness. The pillar is modeled similarly except using segments of equal thickness.

\subsection{Model assumptions}

In this section, we discuss modeling of contact forces. Contact forces include both normal and frictional forces. Any contact forces are assumed to occur between the segments of one body and the joints of the opposing body according to the following rules:

1. If a particular joint or node of one body (joint $i$ ) is located along a perpendicular that emanates from a segment $j$ of the opposing body, a mutual repulsive normal force $N_{i}$ is then generated according to

$$
N_{i}=K_{n}\left(d_{\text {ref }}-d\right)
$$

where $K_{n}$ is the composite normal stiffness of the scale-pillar contact, $d$ is the perpendicular distance between the node and the opposing segment, $d_{\text {ref }}$ is the reference distance at which contact is assumed to first occur, given the finite widths of both the snake scale and the pillar film. We use $K_{n}=0.93 \mathrm{MPa}$ and $d_{\text {ref }}=94 \mu \mathrm{m}$ in our simulations.

2. The line of action of the repulsive force is along the perpendicular (emanating from the segment $j$ ) that contains the joint $i$.

3. We model both static and sliding friction. A mutual tangential force of magnitude $\left|T_{i}\right|$ is generated at the interface between joint $i$ and segment $j$ and acts in a direction to oppose sliding along the segment. If the contact is in a sticking mode, the shear force will be given by

$$
T_{i}=K_{s}\left(d_{s}-d_{s o}\right)
$$


where $d_{s}$ is the combined shear displacement within the interface (i.e., parallel to the local segment that is being contacted) and $d_{s o}$ is the reference contact point where the interface enters the stick mode from the sliding model. While the interface is slipping, the shear force will be given by

$$
T_{i}=\mu N_{i}
$$

For all of the simulations, $\mu$ is set to 0.13 , based on measurements of static friction between the snake scale and the pillar. We assumed that static and kinetic friction coefficients are the same.

\subsection{Equations of motion}

A segment of a snake scale is shown in Fig.1. We define an interior segment as one having two neighbors. The equations of motion include the force balance on each segment as well as the moment balance. Thus, these equations may be written:

$$
\begin{gathered}
m_{i} \mathbf{a}_{i}=-\mathbf{P}_{i}+\mathbf{P}_{i+1}+\left(N_{i}+N_{p i}\right) \hat{\mathbf{n}}_{i}+\left(T_{i}+T_{p i}\right) \hat{\mathbf{t}}_{i}, \\
I_{i} \ddot{\theta}_{i}=\frac{\Delta s}{2}\left(P_{i}^{n}+P_{i+1}^{n}\right)+r_{i} N_{i}+\frac{\Delta s}{2} N_{p i}-k_{i}\left(\theta_{i}-\theta_{i-1}\right)+k_{i+1}\left(\theta_{i+1}-\theta_{i}\right) .
\end{gathered}
$$

Similar equations of motion apply for boundary segments, with only one neighbor. We define the following variables $(\mathbf{F i g}, \mathbf{1})$ ):

- $m_{i}$ is the mass of segment $i$,

- $\mathbf{a}_{i}$ is the acceleration of the center of mass of segment $i$,

- $\mathbf{P}_{i}$ is the force that segment $i$ exerts on segment $i-1$,

- $N_{i}$ is the normal force exerted on scale segment $i$ by a node of the pillar,

- $T_{i}$ is the tangential force exerted on scale segment $i$ by a node of the pillar,

- $N_{p i}$ is the normal force exerted on scale node $i+1$ by a segment of the pillar, 
- $T_{p i}$ is the tangential force exerted on scale node $i+1$ by a node of the pillar,

- $\hat{\mathbf{t}}_{i}$ is the unit tangent parallel to scale segment $i$ and directed toward scale segment $i+1$,

- $\hat{\mathbf{n}}_{i}$ is the normal to scale segment $i: \hat{\mathbf{n}}_{i}=\hat{\mathbf{t}}_{i} \times(\hat{\mathbf{i}} \times \hat{\mathbf{j}})$,

- $I_{i}$ is the mass moment of inertia scale segment $i$ about an axis perpendicular to the plane of the figure and through the center of mass,

- $\theta_{i}$ is the angle that scale segment $i$ makes with the horizontal, measured positive clockwise,

- $\Delta s_{i}$ is the length of scale segment $i$,

- $P_{i}^{n}$ is the normal component of $\mathbf{P}_{i}$,

- $r_{i}$ is the radial distance along scale segment $i$ to the line of action of $N_{i}$,

- $k_{i}$ is the torsional spring constant acting between scale segments $i-1$ and $i$.

Slightly modified versions of the above equations apply to the two end segments of the snake scale. Analogous equations apply to the pillar.

\subsection{Determination of model inputs}

Below we describe how segment mass, mass moment of inertia, local bending moment, and local torsional stiffness are calculated. In consideration of Fig. 2 a, we divide the scale into two major sections: (1) a walled section and (2) a tip section. The walled section extends for $7 \mathrm{~mm}$ from the base, while the tip section has a length of $2.4 \mathrm{~mm}$. For the simulation the walled section consists of 4 equallength segments. Similarly, the tip section consists of 4 equal-length segments. It is important to have a finer resolution (i.e. smaller individual segment lengths) in the tip section to capture the deformation characteristics of the tip. Within each individual segment of the walled section, the cross section is taken as Ushaped with a uniform wall height $h$, uniform wall thickness $t$, uniform base thickness $t$, and uniform base width $b-2 t$. The height of a particular segment 
in the walled section is determined from approximating the shape of the wall as a section of an ellipse along its long direction. Specifically, the height $h_{i}$ of the segment $i$ (for $i$ varying from 1 to 4 ) is given by $h_{i}=h_{1} \sqrt{\left(1-[(i-1) / 4]^{2}\right.}$ where $h_{1}$ is the $3.6 \mathrm{~mm}$, as indicated in Fig.2 $\mathrm{a}$. The cross-sectional area and mass of segment $i$ are then given, respectively, by $A_{i}=2 h_{i} t_{i}+t_{i}\left(b-2 t_{i}\right)$ and $m_{i}=\rho A_{i} \Delta s_{i}$ where $\rho$ is the mass density of the scale $\left(4900 \mathrm{~kg} / \mathrm{m}^{3}\right)$ and $t_{i}$ is assumed to vary linearly from its high value of $0.7 \mathrm{~mm}$ to its low value at the tip of the scale $(0.07 \mathrm{~mm}$ ). In the tip section of the scale (where $i$ varies from 5 to 8$)$, cross-sectional area is given by $A_{i}=h_{i} b_{i}$ where $b_{i}$ is a linearly varying width given by $b_{i}=[1-(i-5) / 4] b$. Based on these segment dimensions in both walled and tip section, the area moment of inertias and mass moment of inertias are computed for each segment of the scale. Corresponding relations are developed for the pillar by dividing it into 8 segments that are uniform in all dimensions.

To obtain the local torsional stiffness, we use the relationship between bending moment and curvature for a flexible beam:

$$
M(s)=E I_{A}(s) \frac{d \theta}{d s}
$$

where $M(s)$ is the local bending moment, $s$ is a measure of arc length, $E$ is the modulus of elasticity, $I_{A}$ is the local area moment of inertia of the cross section, and $\theta$ is the angle that the beam centerline makes with the horizontal. The moment experienced by segment $i$ in accordance with a small difference in inclination between it and the preceding segment, is given by

$$
M_{i}=\frac{E I_{A_{i}}}{\Delta s_{i}}\left(\theta_{i}-\theta_{i-1}\right) .
$$

Thus, the location torsional stiffness becomes $k_{i}=E I_{A_{i}} /\left(\Delta s_{i}\right)$.

\subsection{Integration of equations of motion}

We simulate the interaction between a snake scale and pillar with respect to time. The snake scale is initially oriented at a prescribed angle with respect 
to the horizontal and its base point is translated along a prescribed path at a constant horizontal speed. The path of the base point is determined from the video images from the experiment. Additionally, the initial configuration of the pillar is based on the first video frame that shows scale-pillar engagement.

At any instant, specification of the snake scale base position along with all angular positions and angular velocities of both bodies results in three unknown quantities for each segment of either body. All other quantities are determined directly from body positions and body velocities. At the same time, as per the equations presented above, there are three independent equations for each segment. Hence, at each time step, all of the unknowns can be determined by solving the associated system of equations. In the present work, we use the well-known 4th-order Runge-Kutta integration scheme [12].

\subsection{Validation of model}

The first step in our study is verification of our numerical model for studying the dynamic interaction of two elastic beams. For this purpose we compare the results of the dynamic numerical simulation to two different analytical models. The test problem is one for which an originally vertical, cantilevered pillar is loaded horizontally via prescribed force at its end. In the numerical simulation, the pillar is allowed to attain equilibrium asymptotically over time. The resulting configuration is compared to the analytical solution from Euler-Bernoulli

beam theory and to the analytical static equilibrium solution associated with the representation of the pillar as series of finite segments coupled by torsional springs (as in the dynamic simulation). As shown in Fig. 3 , there is excellent agreement among the three models. In doing the comparison, it should be noted that a special technique was applied to the discrete, segment-based models to mimic the zero-slope cantilever boundary condition. That is, the stiffness of the base torsional spring was set in relation to the value of the stiffness at all other joints ( $k=E b h^{3} / 12 \Delta s$, from above) so that the slope at the base node, as estimated via a 2nd-order finite difference method applied to the first three nodal points, would be zero. 
In order to calibrate the pillars we attach a stiff glass arm to the end of a weight micro scale and put the pillar array on a micrometer platform. Placing the tip of the glass arm close to the tip of the film and adjusting the platform dial, a force-displacement curve is made for the pillar (Fig. 3 b). As shown in this figure, increasing the contact height, $a$, decreases the slope of forcedisplacement curve. The horizontal force is seen to increase more-or-less steadily with increasing glass displacement, but with a decreasing rate as the tip of the glass arm slides toward the tip of the pillar. Ultimately, at certain displacement, the glass arm slides past the tip so that contact is lost. As illustrated in Fig. $\mathbf{3}$ b, there is a reasonably good match between the experimental data and the results of the numerical simulation, particularly for the larger value of contact height, $a$.

\subsection{Measurement of scale torsional stiffness}

We assessed the torsional stiffness of a belly scale by interacting a single scale with a glass arm attached to a load-cell. A glass arm extends vertically from the load-cell and engages a belly scale while the (conscious) snake is supported and translated laterally. The load-cell records the horizontal force exerted upon the glass arm by the load-cell. Results of this type of measurement for four different belly scales are shown in Table 2. Based on the analysis of the video stills, we developed measurements of the overall orientation of the snake belly

255 ("belly angle"), $\theta_{b}$, the scale opening angle with respect to the belly $\theta_{o}$, and the scale opening angle with respect to the horizontal, $\theta$. Note that a positive value for the belly angle means that the head-side is lower than the tail-side. Note also that $\theta=\theta_{o}-\theta_{b}$.

We also present in the table the magnitude of vertical force, $|W|$, acting on the load-cell arm and the horizontal force, $F$, as well as their moment arms $d_{1}$ and $d_{2}$, respectively, relative to the base of the scale. Now the value of the horizontal force comes directly from the load-cell, whereas the magnitude of vertical force is assumed to be $0.29 F$, where 0.29 is the measured kinetic friction coefficient between the snake scale and the glass load-cell arm. Based 
on these data, we compute the torsional spring constant at the base of the scale (viewing the scale here as a passive torsional spring). That is, the computed spring constant is given by

$$
k=\frac{F d_{2}-W d_{1}}{\theta}
$$

In the second to last column, $k$ is computed under the assumption that the

260 assuming that the scale is sliding down the load-cell arm. Under the assumption of upward slip, the average value of torsional spring constant is $10.5 \mathrm{~N} . \mathrm{mm} / \mathrm{rad}$, whereas under the assumption of downward slip, the computed spring constant is 6.0 N.mm/rad.

While it is known, from the video record, that the overall motion of scale contact point is upward along the load-cell arm, there is the possibility that, at the instant of the force measurement, the scale is in a stick mode rather than in a sliding mode. In this case, the vertical force $W$ could be anywhere in between $-0.29 F$ (for upward slip) and $0.29 F$ (for downward slip). Thus, without knowing that state of sticking or slipping, we can conclude only that spring constant is within the value for sliding up or sliding down. Therefore, if there is a single value of spring constant that is consistent with all of the four video frames, it must be simultaneously within each of the four ranges delineated by the values for sliding up and sliding down in Table 2. Therefore, $k$ must be between 7.21 and 8.36 N.mm/rad.

\subsection{Simulation of scale torsional stiffness measurement}

To provide validation of the process to determine the torsional stiffness, as outlined above, we conduct a simulation of the engagement of a snake scale with a load-cell arm. The load-cell arm is modeled as a rigid, vertical member coupled to its base via a torsional spring. The load-cell's torsional spring constant is determined from the video record by comparing the moment caused by the horizontal force to the angular deflection of the load-cell arm (with an 
uncertainty of approximately $+/-100 \mathrm{~N} . \mathrm{mm} / \mathrm{rad}$ ). This procedure results in a computed value of 1250 N.mm/rad for the torsional spring constant of the load-cell. Additionally, based on the video record, the tip of the scale is observed to engage the load-cell arm at approximately $30 \mathrm{~mm}$ above the base of the load-cell (with an uncertainty of about $+/-0.5 \mathrm{~mm}$ ). Thus, there are three measured parameter values that are targeted for matching via the simulation: (1) a contact point height between 29.5 and $30.5 \mathrm{~mm},(2)$ the measured opening angle of the scale, and (3) the measured horizontal force. In trying to match these experimental values, three input parameters are varied: (1) the vertical position of the scale base (relative to the base of the load-cell), (2) the horizontal position of the scale base (relative to the location of the load-cell), and (3) the scale torsional spring constant. Results of this simulation are shown in Table 3. As observed, good matching with the experimental findings is obtained for values of torsional spring constant that range between 7 N.mm/rad and 9.5 N.mm/rad. This range of torsional spring constants is close to the range found in $\S 3.7$. It is concluded, therefore, that the torsional spring constant for the base scale is in the vicinity of $8 \mathrm{~N} . \mathrm{mm} / \mathrm{rad}$. Note that the above analysis treats the scale as a passive torsional spring. That is, we are supposing that, for this test, the snake is not actively controlling its scales. The fact that we obtain a fairly small range for the scale torsional spring constant across the four different scales which are measured with opening angles that ranged from 45 deg to 63.3 deg provides some support for this notion.

\section{Results}

We perform experiments of the snakes sliding against our pillar arrays. In each trial, we drag the snake over the pillars resulting in several engagements of the snake scales with the pillars. Fig. 4 a shows one of the interactions of a snake scale with a pillar. This process which occurs in 0.13 seconds, and involves a total body displacement of $3.4 \mathrm{~mm}$. The first frame shows the initial contact between the scale and pillar. Then the scale slides down the pillar as 
the pillar begins to deflect. In the second frame the contact point between scale tip and obstruction is further down on the pillar. Note the large opening angle of the snake scale. In the last frame, the scale opening angle has grown even larger, but the contact point is now further up the pillar. Note that last frame represents the last image that we can observe the scale in contact with the pillar. Fig 4 b,c show snapshots of the computer simulation corresponding to each of the displayed video frames with and without applying an opening moment at the base of the scale. When no opening moment is applied the scale is passively deformed; a scale with moment refers to a scale that includes an active contribution to the response during engagement with the pillar. As seen in Fig. $\mathbf{4}$, the simulation with an applied base moment does a good job of capturing the essential features of the interaction, whereas, in the absence of an applied moment (Fig, 4 ), the scale quickly slides up the pillar (note the small base displacement that has occurred in Fig. 4 c by the time the scale has reach the top of the pillar).

Additional evidence for the role of active mechanisms comes from comparing scale-pillar engagement results between a conscious and unconscious snake. First, it is noted that when the snake, whether conscious or unconscious, is dragged backward across the pillar array, most of the scales do not engage, but rather, simply skim over the top of the pillars. That is the scales tend to remain fully closed, each keeping flush contact with its adjacent anterior scale. However, in certain cases, a scale will not be flush as it approaches a pillar, having its tip lower than the top of pillar. In analyzing the video record, we observe several such engagements. Of particular interest are the set of cases where the angle of approach is shallow, because, based on our simulation, a purely passive response leads to only sliding up the pillar as opposed to sliding down first and then eventually up. Results for the unconscious snake shows that there are 7 engagements where the opening angle is less than $7^{\circ}$, none of which slides down. 340 By comparison, for the conscious snake, there were 8 such engagements with opening angles less than $7^{\circ}, 3$ of which slide down.

We estimate the horizontal contact force between scale and pillar by consid- 
ering various directions of sliding that could be occurring in our sparse video frames. Assuming that the beginning and middle frames correspond to the scale sliding down the pillar, while the end frame corresponds to the scale sliding up, we compute horizontal force values of $0.16 \mathrm{~N}, 0.35 \mathrm{~N}$ and $0.5 \mathrm{~N}$, respectively for the first, middle and last frame in Fig $\sqrt{4}$, respectively. Since this horizontal force resists the backward horizontal translation of the snake, it represents, in the context of the snake slipping or tending to slip backwards, the friction force between the snake and the counter surface. This range of force is around 6 percent of the snake's body weight. Thus, a snake need to only have 16 scales in contact with the pillars to prevent sliding. This scenario is quite feasible, considering the corn snake in our study has more than 100 scales.

This method of preventing sliding thus is quite different from the sliding forces in Amonton's classical friction laws. Specifically, the snake's resistance to sliding parallel to a surface, does not rely on the presence of any appreciable normal force. Thus, this method of preventing sliding can be used for climbing vertical surfaces such as tree trunks.

In addition to our video sequences, we also provide quantitative comparisons between our model and experiment. We consider three variables that characterize the motion of the scale (Fig $[\mathbf{5}$ ). Fig, 5 shows the scale opening angle, scale-pillar overlap, and pillar tip deflection for experimental trials 1 (Fig, $\mathbf{5}$ b,c) and 2 (Fig. 5 d,e). These trials are two scale-pillar interactions (out of many interactions observed in our experiments) that we analyze in detail. As observed, there is reasonable agreement in $\mathbf{F i g}, \mathbf{5 b}$,d. However, it is important to note that this degree of agreement was obtained by introducing two modifications to the basic model of the snake scale. First, it is found, based on analyzing the initial contact configuration (i.e., per the beginning video frame above), the contact forces initially exert a closing moment on the scale. Fig. $\mathbf{6}$ shows, schematically the geometry of a typical contact at initial engagement. For the scale to be on the verge of sliding down the pillar, the friction force $T$ would be tangent to the pillar and pointing upward. The combination of the normal force $N$ and the friction force cause the line of action to pass to the right of the base of the scale, 
thereby exerting a moment that tends to close the scale. Hence, if the scale base angle is modeled as a passive torsional spring, the scale will tend to close at the beginning of engagement and slides up the pillar (Fig, 5 ;,e). Thus, to achieve a level of agreement with experiment, we assume that the snake actively exerts a constant opening moment on the scale throughout the engagement. This condition is equivalent to assuming a particular value for the equilibrium base angle, namely 14 degrees. Additionally, we set the torsional spring constant to be 4 N.mm/rad, which is less than that found above through load-cell measurement (approximately 7-9 N.mm/rad). In order to further clarify the choice of these two parameters we conduct a simulation to find maximum horizontal displacement as a function of scale base torsional stiffness and free angle of scale 385 base. As shown in Fig,7, torsional spring constant of $4 \mathrm{~N} . \mathrm{mm} / \mathrm{rad}$ and the equilibrium base angle of 14 degrees result in the highest horizontal displacement before disengagement of scale and pillar. Small free angles result in sliding up and thus disengagement before any horizontal displacement of snake scale occurs. Base torsional stiffnesses larger than 4 N.mm/rad also result in small maximum horizontal displacements.

The role of active opening moment is further illustrated via Fig.4 which shows the simulation results when we assume that the snake scale is a purely passive torsional spring with a spring constant of $5.12 \mathrm{~N} . \mathrm{mm} / \mathrm{rad}$ (the lowest value found in Table 22. As observed, the scale slides up the pillar right away and begins to lose contact when the base of the scale has displaced less than 0.1 $\mathrm{mm}$. In fact, the scale is found always to slide up and off of the pillar, from the initial configuration, no matter what value of scale base torsional spring constant is used, unless one introduces an opening moment as done above $(\mathbf{F i g}, 4 \mathrm{~b}$ and Fig, 5 , d).

Comparing the experimental measurements and simulation results presented in this study, it is inferred that snakes can tune the torsional stiffness of their ventral scales to adapt to the environment and attach more effectively to their substrates. We hypothesize snakes can use their ventral cutaneous musculature to actively control their ventral scales torsional stiffness [13]. This observation 
unconscious snakes [8]. However, systematic experiments (using an apparatus that minimizes human handling of the snakes in these measurements) in addition to using larger sample of animals are needed to strongly support this hypothesis.

\section{Conclusions}

In this study, we investigate the resistive force of a single snake scale. We develop a model for the snake scale engagement with a flexible pillar and compare its results to video recordings of snakes' bellies being pulled manually over an array of pillars. A force and deformation analysis performed in conjunction with the videography suggests that snakes can actively control their ventral scales to achieve prolonged contact and greater resistance with the pillar. The idea of controlling frictional properties as a function of position and time could result in developing new functional surfaces for making effective interactions with complex environment and reducing energetic cost of locomotion.

\section{Acknowledgements}

The authors thank Chris Kingsbury for helping with experiments, Tim Nowak and Candler Hobbs for photography, and the NSF (PHY-0848894) as well as the Elizabeth Smithgall Watts endowment for financial support.

\section{References}

[1] A. Filippov, S. N. Gorb, Frictional-anisotropy-based systems in biology: structural diversity and numerical model, Scientific reports 3.

[2] J. Hazel, M. Stone, M. Grace, V. Tsukruk, Nanoscale design of snake skin for reptation locomotions via friction anisotropy, Journal of Biomechanics 32 (5) (1999) 477-484. 
[3] M. Scherge, S. Gorb, S. Gorb, Biological micro-and nanotribology: nature's solutions, Springer Verlag, 2001.

[4] R. Berthé, G. Westhoff, H. Bleckmann, S. Gorb, Surface structure and frictional properties of the skin of the amazon tree boa corallus hortulanus (squamata, boidae), Journal of Comparative Physiology A: Neuroethology, Sensory, Neural, and Behavioral Physiology 195 (3) (2009) 311-318.

[5] M. J. Benz, A. E. Kovalev, S. N. Gorb, Anisotropic frictional properties in snakes, in: SPIE Smart Structures and Materials+ Nondestructive Evaluation and Health Monitoring, International Society for Optics and Photonics, 2012, pp. 83390X-83390X.

[6] J. Gray, H. W. Lissman, The kinetics of locomotion of the grass-snake, J. Exp. Biol. 26 (1950) 354-367.

[7] H. Abdel-Aal, M. El Mansori, S. Mezghani, Multi-scale investigation of surface topography of ball python (python regius) shed skin in comparison to human skin, Tribology letters 37 (3) (2010) 517-527.

[8] H. Marvi, D. Hu, Friction enhancement in concertina locomotion of snakes, Journal of the Royal Society Interface 9 (76) (2012) 3067-3080.

[9] D. Hu, J. Nirody, T. Scott, M. Shelley, The mechanics of slithering locomotion, Proceedings of the National Academy of Sciences 106 (25) (2009) 10081.

[10] H. Marvi, J. Bridges, D. Hu, Snakes mimic earthworms: propulsion using rectilinear traveling waves, Journal of the Royal Society Interface 10 (84).

[11] H. Marvi, G. Meyers, G. Russell, D. Hu, Scalybot: a snake-inspired robot with active frictional anisotropy, in: ASME, Dynamic Systems and Control (DSCC) (2011).

[12] S. C. Chapra, R. P. Canale, Numerical methods for engineers, Vol. 2, 455 McGraw-Hill, 2012. 
[13] C. Gans, T. S. Parsons, A. d. Bellairs, Biology of the Reptilia, Vol. 1, Academic Press, 1969. 


\begin{tabular}{|c|c|c|}
\hline Parameter & Scale & Transparency \\
\hline$L$ & $9.4 \mathrm{~mm}$ & $4 \mathrm{~mm}$ \\
\hline$b$ & $25 \mathrm{~mm}$ & $24.2 \mathrm{~mm}$ \\
\hline$h_{0}$ & $0.7 \mathrm{~mm}$ & $0.12 \mathrm{~mm}$ \\
\hline$h_{e}$ & $0.07 \mathrm{~mm}$ & $0.12 \mathrm{~mm}$ \\
\hline$E$ & $400 \mathrm{MPa}$ & $2280 \mathrm{MPa}$ \\
\hline
\end{tabular}

Table 1: Input parameters: length $L$, width $b$, thickness at base $h_{0}$, thickness at tip $h_{e}$, and stiffness $E$ for scale and transparency.

\begin{tabular}{|c|c|c|c|c|c|c|c|c|c|}
\hline & & & & & & & & Sliding Up & Sliding Down \\
\hline Scale & $\theta_{b}$ (deg) & $\theta_{0}$ (deg) & $\theta$ (deg) & $F(N)$ & $d_{2}(m m)$ & $|W|(N)$ & $d_{1}(m m)$ & $\mathrm{k}(\mathrm{N} \cdot \mathrm{mm} / \mathrm{rad})$ & $\mathrm{k}(\mathrm{N} \cdot \mathrm{mm} / \mathrm{rad})$ \\
\hline 1 & 2.5 & 45.5 & 43 & 1.13 & 6.41 & 0.33 & 6.87 & 11.96 & 6.29 \\
\hline 2 & 12.5 & 62.8 & 50.3 & 1.02 & 7.23 & 0.30 & 6.00 & 8.36 & 5.12 \\
\hline 3 & 19.3 & 63.3 & 44 & 1.34 & 6.53 & 0.39 & 6.76 & 10.31 & 5.55 \\
\hline 4 & 2.1 & 53.9 & 51.8 & 1.19 & 7.39 & 0.35 & 5.81 & 11.48 & 7.21 \\
\hline
\end{tabular}

Table 2: Estimations of scale base spring stiffness based on load-cell measurements. Kinetic coefficient of friction, $\mu$, between snake and glass is 0.29 . Horizontal contact force (normal force) is $F$ and magnitude of friction is $|W|=\mu F$. For sliding up, friction force on scale contributes to the opening moment caused by the horizontal force. For sliding down, friction force opposes the opening moment caused by the horizontal force. $\theta_{b}$ is the belly angle, $\theta_{0}$ is base opening angle, $\theta$ is base angle with respect to the horizontal, $k$ is the scale base torsional spring value, and $d_{1}$ and $d_{2}$ are the moment arms for $|W|$ and $F$, respectively. 


\begin{tabular}{|c|c|c|c|c|c|c|}
\hline \multirow{2}{*}{ Scale } & \multirow{2}{*}{$\theta_{b}(\mathrm{deg})$} & \multirow{2}{*}{$\begin{array}{c}\mathrm{k}(\mathrm{N} . \mathrm{mm} / \mathrm{rad}), \\
\text { Simulation }\end{array}$} & \multicolumn{2}{|c|}{$\theta_{\mathrm{o}}$ (deg) } & \multicolumn{2}{|c|}{$F(N)$} \\
\cline { 4 - 7 } & & & Experiment & Simulation & Experiment & Simulation \\
\hline 1 & 2.5 & 9.5 & 45.5 & 45.2 & 1.13 & 1.14 \\
\hline 2 & 12.5 & 7 & 62.8 & 63.5 & 1.02 & 1.04 \\
\hline 3 & 19.3 & 8 & 63.3 & 63.8 & 1.34 & 1.35 \\
\hline 4 & 2.1 & 9 & 53.9 & 53.4 & 1.19 & 1.15 \\
\hline
\end{tabular}

Table 3: Experimental data versus simulation results for horizontal contact force, $F$, and base opening angle, $\theta_{0}$. There is a good agreement for both $F$ and $\theta_{0}$ in each case with a scale base torsional spring value, $k$ that ranges between 6.6 and $8.8 \mathrm{~N} . \mathrm{mm} / \mathrm{rad}$. 

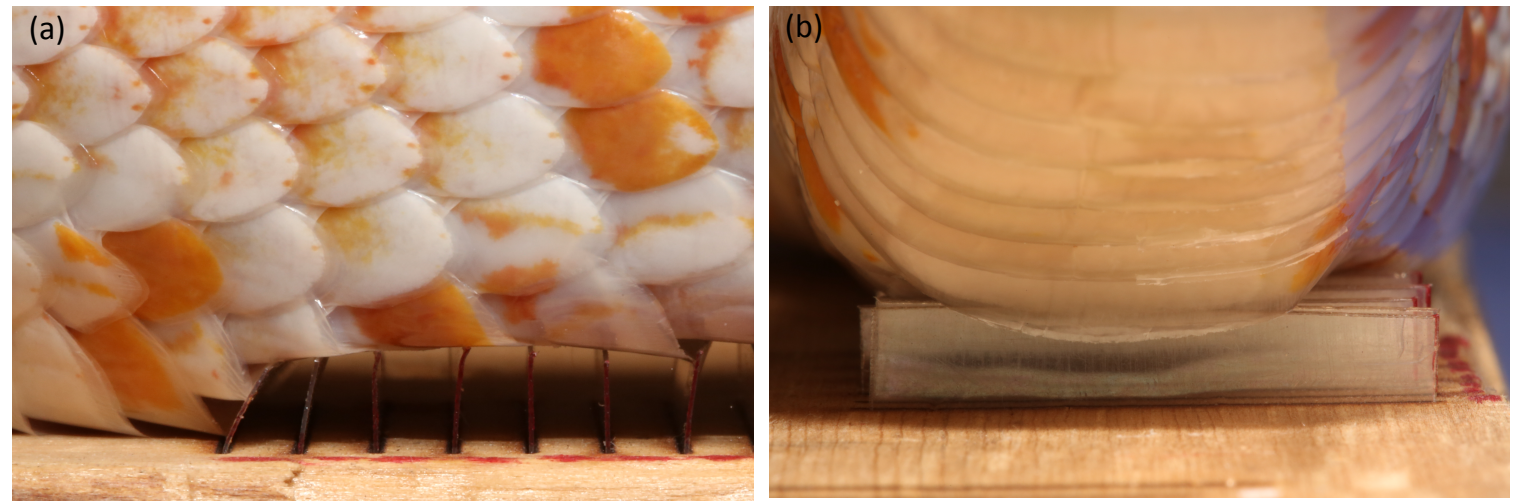

(c)

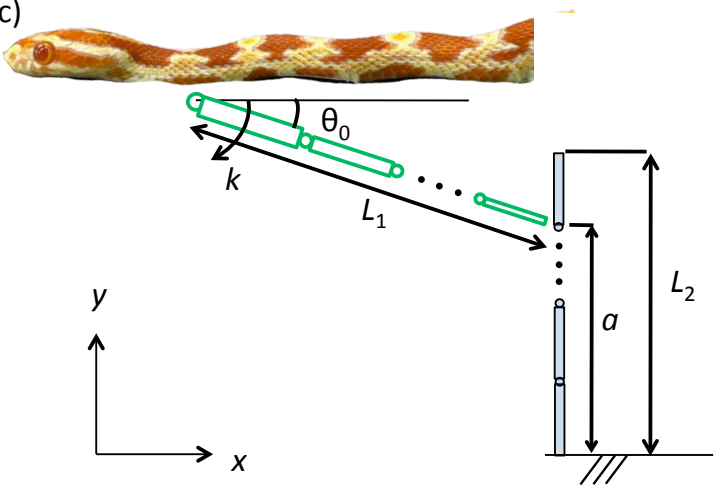

(d)

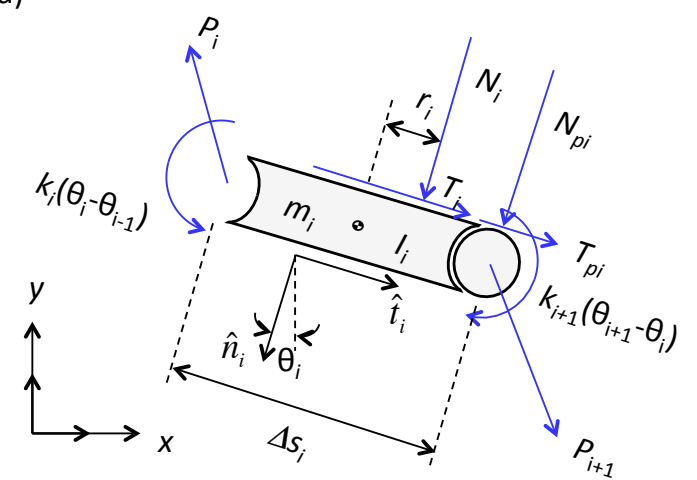

Figure 1: Snake scale interacting with an array of pillars: (a) side view, (b) front view, and (c) schematic. Each segment is coupled to adjacent segments via torsional springs (depicted by circles at junctions). The solid black circles are a representation of additional links that are not shown in this schematic. $L_{1}$ and $L_{2}$ are scale and pillar lengths, respectively. $\theta_{0}$ is initial scale base angle, $k$ is scale base torsional stiffness, and $a$ is height of contact. (d) Free body diagram of a segment. Blue arrows show forces and moments applied. $m_{i}$ is the mass of segment $i, \mathbf{a}_{i}$ is the acceleration of the center of mass of segment $i, \mathbf{P}_{i}$ is the force that segment $i$ exerts on segment $i-1, N_{i}$ is the normal force exerted on scale segment $i$ by a node of the pillar, $T_{i}$ is the tangential force exerted on scale segment $i$ by a node of the pillar, $N_{p i}$ is the normal force exerted on scale node $i+1$ by a segment of the pillar, $T_{p i}$ is the tangential force exerted on scale node $i+1$ by a node of the pillar, $\hat{\mathbf{t}}_{i}$ is the unit tangent parallel to scale segment $i$ and directed toward scale segment $i+1, \hat{\mathbf{n}}_{i}$ is the normal to scale segment $i, I_{i}$ is the mass moment of inertia scale segment $i$ about an axis perpendicular to the plane of the figure and through the center of mass, $\theta_{i}$ is the angle that scale segment $i$ makes with the horizontal, measured positive clockwise, $\Delta s_{i}$ is the length of scale segment $i, r_{i}$ is the radial distance along scale segment $i$ to the line of action of $N_{i}$, and $k_{i}$ is the torsional spring constant acting between scale segments $i-1$ and $i$. 

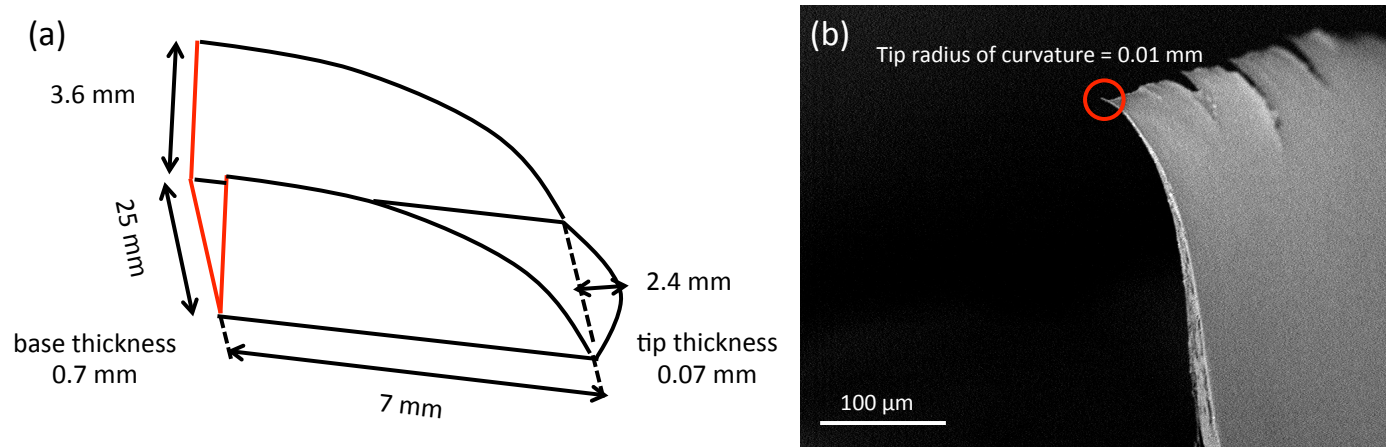

Figure 2: (a) schematic and (b) SEM of a ventral scale. The red lines in the schematic represent the parts of the scale that are attached to the snake body. The total length of the scale used in our simulations (base to tip) is $9.4 \mathrm{~mm}$. The dashed line separates the walled section from the tip section. The primary surface shown in the SEM image is the dorsal side of the scale. We cut this scale in half (longitudinally) to see the cross section of this scale. 
(a)

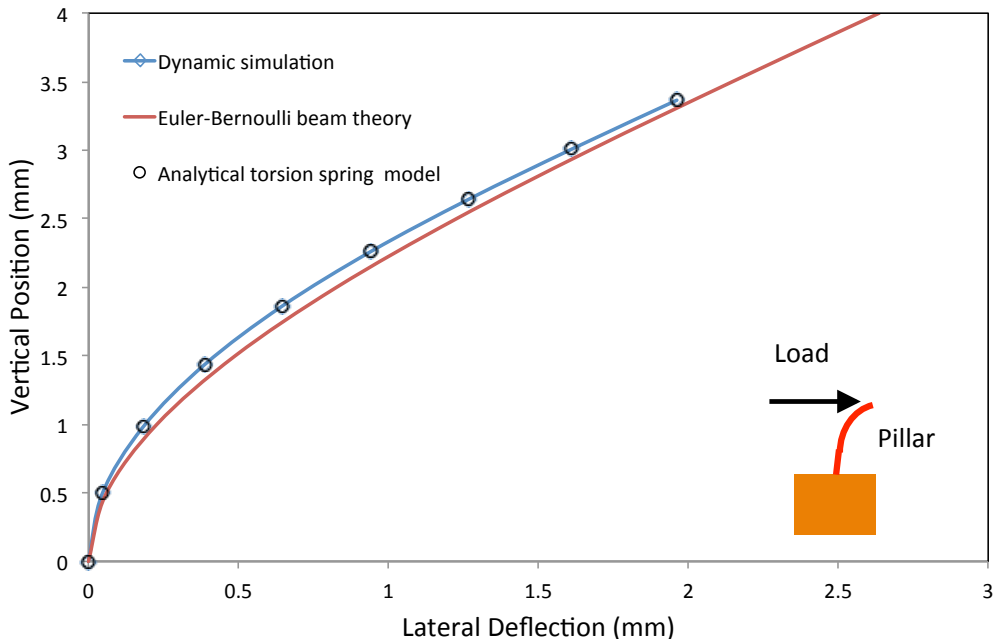

(b)

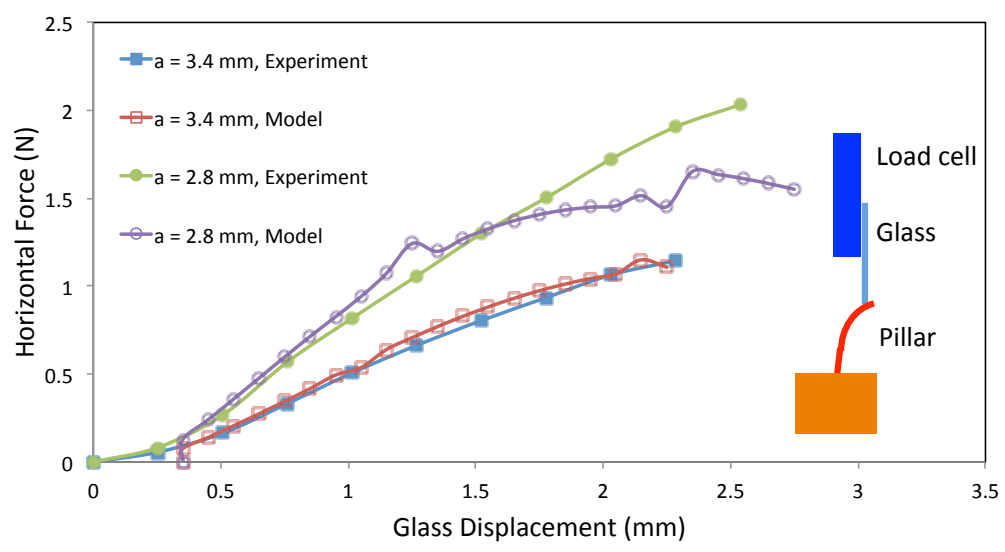

Figure 3: (a) Lateral deflection versus vertical position of the beam under $1 \mathrm{~N}$ load obtained from dynamic simulation, Euler-Bernoulli theory, and analytical torsion spring model. The inset illustrates the loading configuration. (b) Interaction of glass and a pillar at two different contact heights, $a$. The plot illustrates horizontal force as a function of horizontal displacement of pillar where it makes a contact with glass. The inset shows the schematic of this experiment. 
(a)
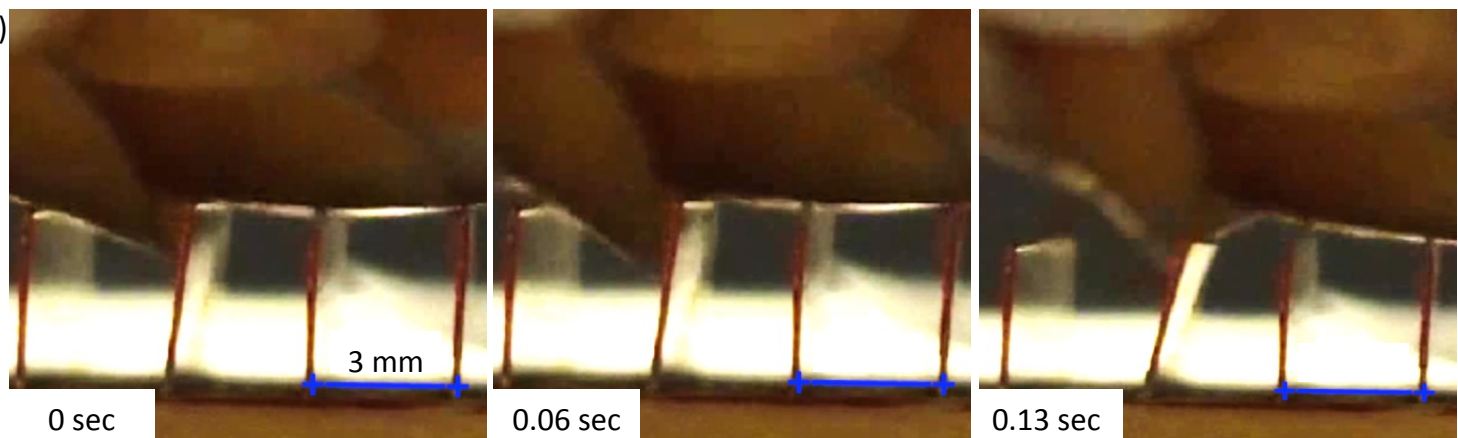

(b)
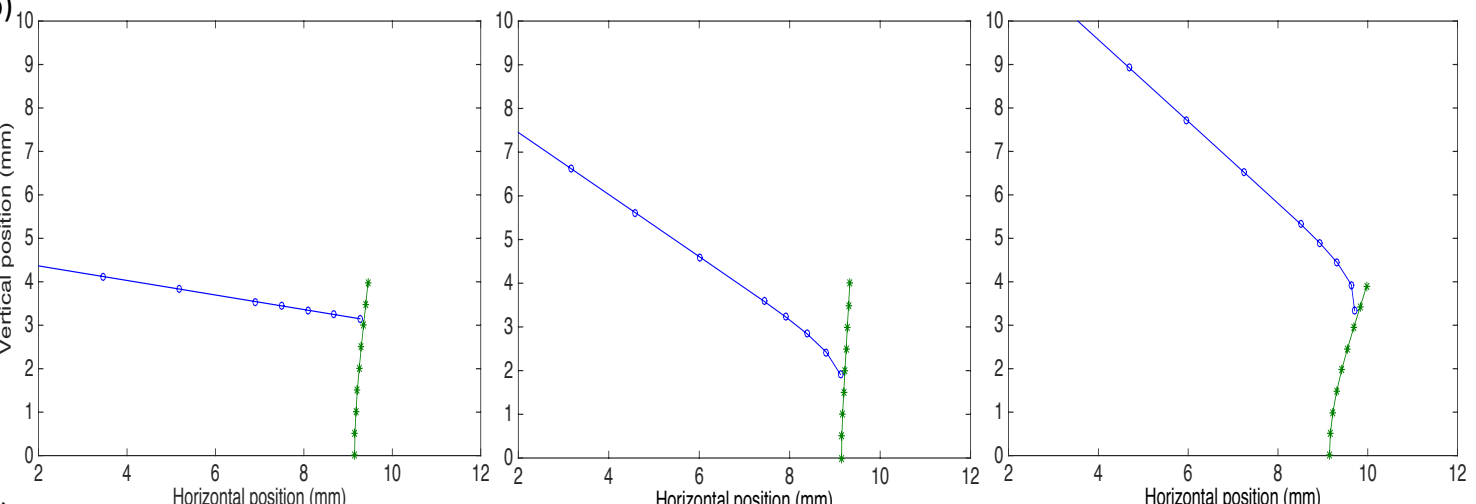

(c)
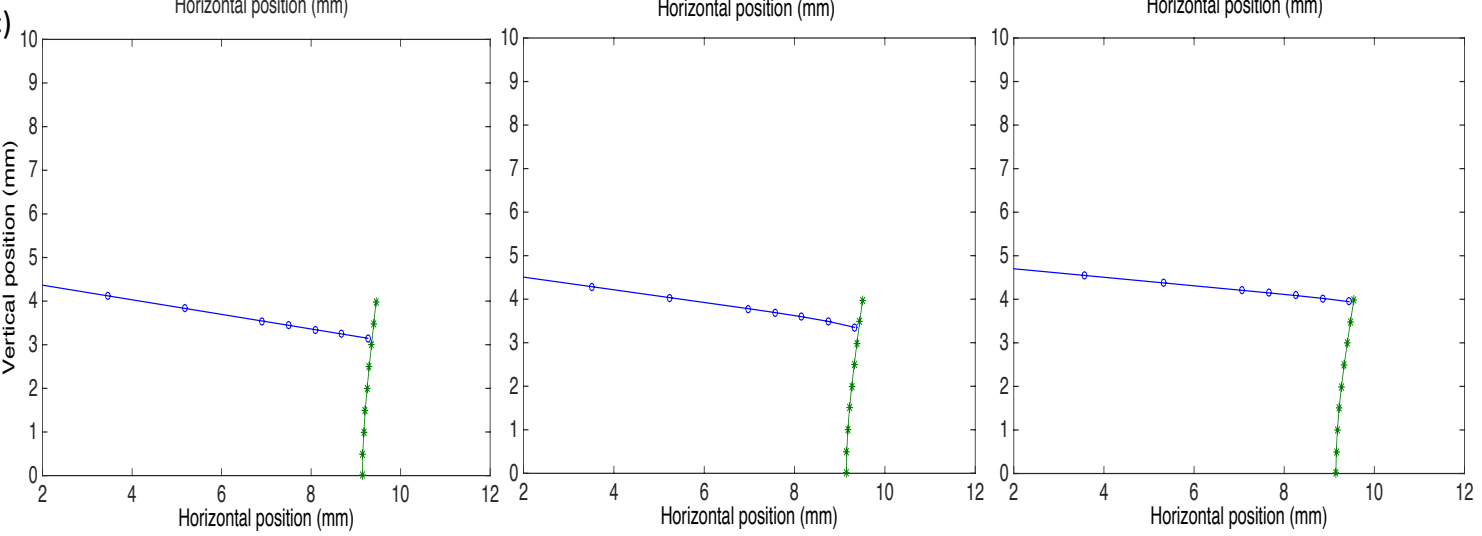

Figure 4: Video sequence of a snake scale interacting with a pillar. (a) Experiment versus model (b) with and (c) without applied base moment. The scale in panel (c) slides up due to the lack of applied opening moment at its base. 

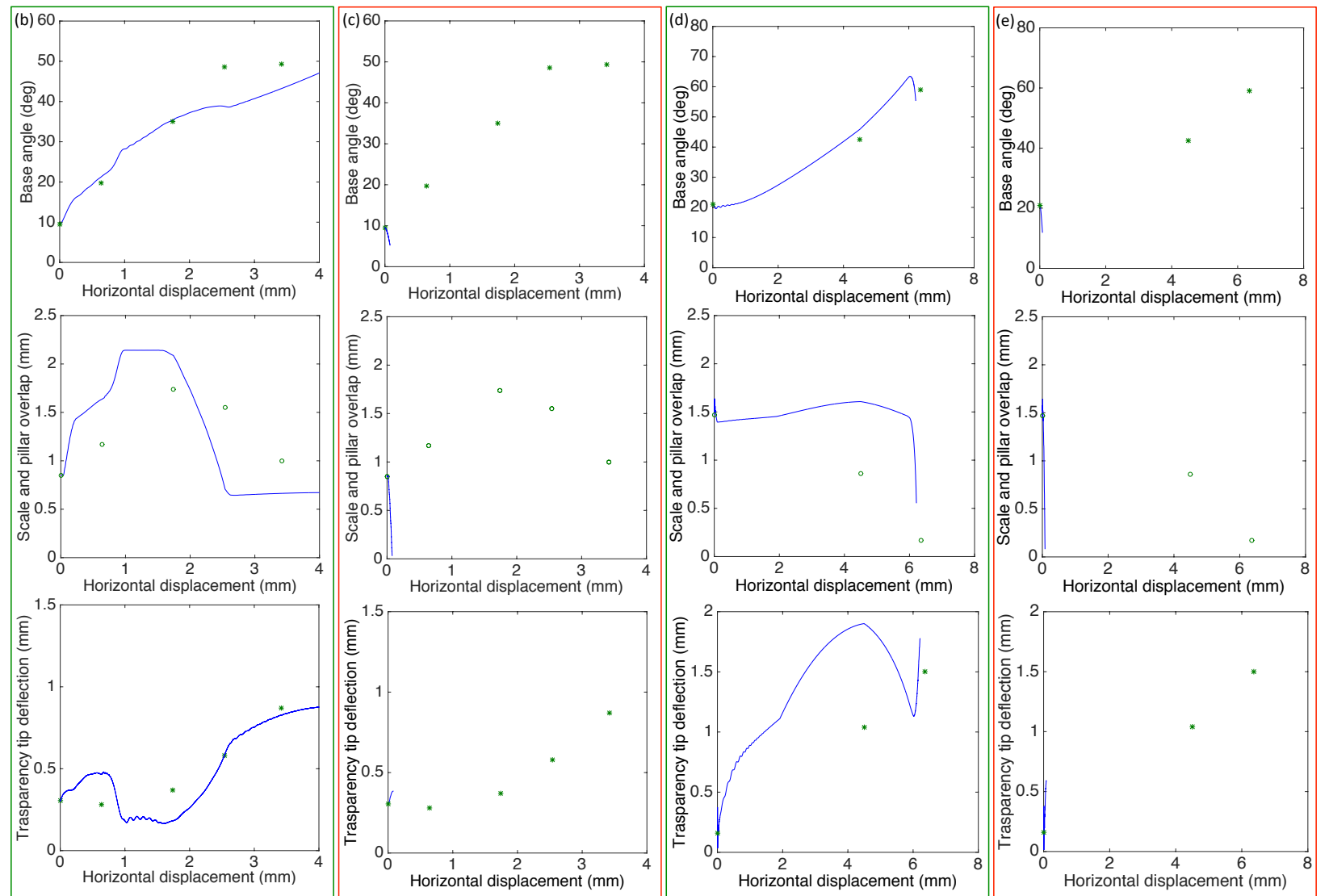

Figure 5: (a) Schematic of scale-pillar interaction. Comparison of simulation (continuous curve) and experiment (symbols) for base angle, scale and pillar overlap, and transparency tip deflection as a function of snake horizontal displacement for (b) trial 1, with applied base moment, (c) trial 1, without applied base moment, (d) trial 2, with applied base moment, and (e) trial 2, without applied base moment (trials 1 and 2 are two of the experimental scale-pillar interactions that are analyzed in detail). For simulations with applied base moment, the base spring stiffness is set to $4 \mathrm{~N} . \mathrm{mm} / \mathrm{rad}$ and the equilibrium base angle is set to 14 degrees. 


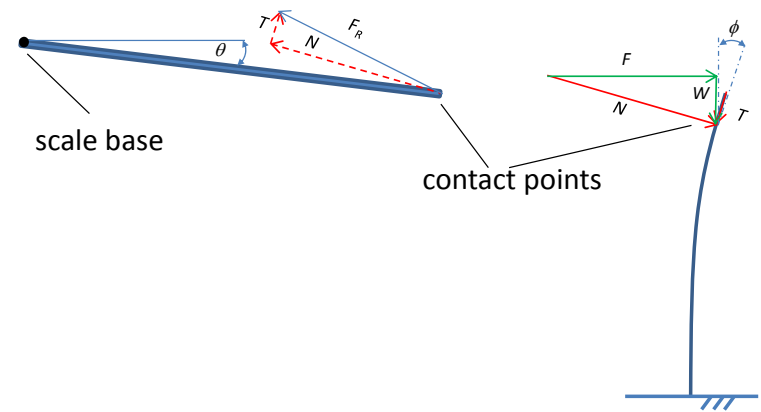

Figure 6: Contact forces between scale tip and flexible pillar for a case that the scale is sliding or tending to slide down the pillar (scale and pillar are isolated here to better illustrate forces).

Resultant contact force, $F_{R}$, on scale tip causes a closing moment on the scale for the given contact configuration. $T$ and $N$ are tangential and normal components of the contact force.

$F$ and $W$ are horizontal and vertical components of the contact force. $\theta$ is the scale base angle and $\phi$ is the pillar tip deflection angle. 

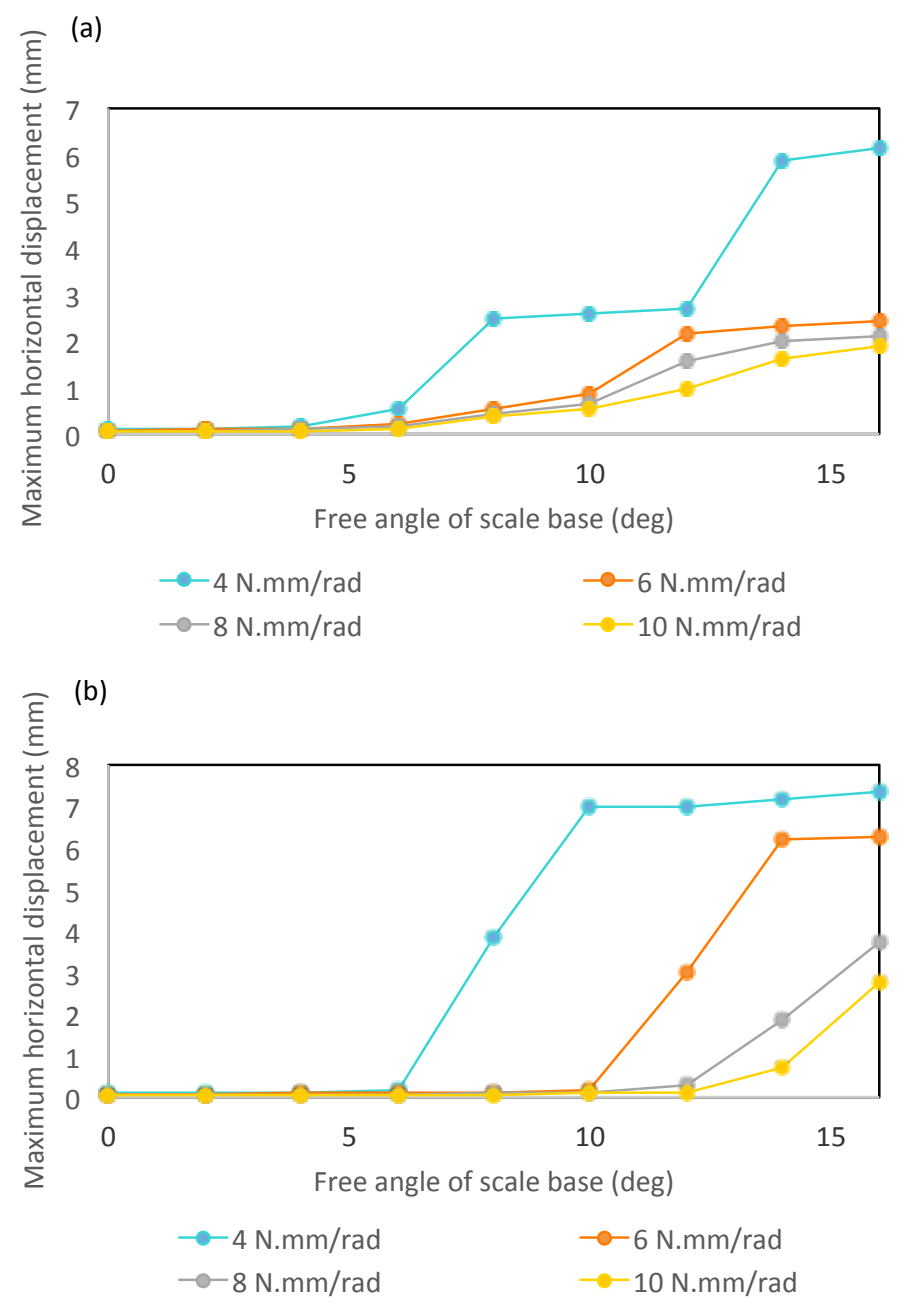

Figure 7: Simulation results for maximum horizontal displacement as a function of free angle of scale base and base torsional stiffness for parameters according to (a) experimental trial 1 and (b) experimental trial 2. 